\title{
Acometimento cardíaco em pacientes com doença de Chagas aguda em microepidemia familiar, em Abaetetuba, na Amazônia Brasileira
}

\author{
Cardiac attacks in patients with acute Chagas' disease in microepidemic \\ familiar episode, in Abaetetuba City, Brazilian Amazon
}

\section{Ana Yecê das Neves Pinto', Geraldo Saburo Harada ${ }^{2}$, Vera da Costa Valente ${ }^{1}$, José Elson Araújo Abud ${ }^{1}$, Francisco dos Santos Gomes ${ }^{1}$ Gilberto César Rodrigues de Souza ${ }^{1}$ e Sebastião Aldo da Silva Valente ${ }^{1}$}

\begin{abstract}
Resumo Os autores mostram os principais achados clínicos relativos ao acometimento cardíaco, em pacientes portadores de doença de Chagas aguda em mais um episódio de microepidemia familiar na Amazônia brasileira. Foram estudados 13 pacientes com doença de Chagas aguda, procedentes do município de Abaetetuba-PA e submetidos à avaliação clínica e cardiológica, eletrocardiograma e ecocardiograma. As extra-sístoles supraventriculares e/ou ventriculares ocorreram em 38,5\% dos casos. Bloqueios de ramo direito e bloqueios átrio-ventriculares de $1^{\circ}$ e $2^{\circ}$ graus, foram encontrados em $30,8 \%$ dos doentes. Chamam atenção dois achados no ecodopplercardiograma: derrame pericárdico e imagem sugestiva de formação aneurismática em dois pacientes respectivamente. Os achados revelam comprometimento cardíaco agudo, com evidências de miocardiopatia e alterações no sistema de condução do coração, havendo similaridade com a descrição da doença em áreas endêmicas.
\end{abstract}

Palavras-chaves: Cardiopatia chagásica aguda. Doença de Chagas. Amazônia.

\begin{abstract}
The authors describe the main clinical findings relative to cardiac involvement, in patients with acute Chagas' disease (CD) in yet another familial micro-epidemic episode of $C D$ in Amazon region. Thirteen patients were studied with acute Chagas' disease, resident in the city of Abaetetuba in Pará State; they were submitted to clinical and heart evaluation, with electrocardiograph and echocardiograph exams. Ventricular extrasystole occurred in 38.5\% of the cases. Right bundle branch block and 1st and 2nd degree atrioventricular block were found in $30.8 \%$ of the patients. Attention is called to two findings in the Doppler echocardiography: pericardiac involvement and an image suggestive of aneurismatic formation in two patients. The findings reveal acute heart disease, with evidence of cardiomyopathy and alterations in the conduction system of the heart, bearing similarity with the description of the disease in endemic areas.
\end{abstract}

Key-words: Acute chagasic cardiopath. Chagas' disease. Amazon.

Em áreas endêmicas de doença de Chagas (DC), a manifestação aguda da doença tem se tornado infreqüente e pouco diagnosticada, não só em virtude das medidas de controle impostas pelos programas bem estruturados de combate à transmissão vetorial ${ }^{17}$, como também pela própria história natural da mesma, em que a apresentação sintomática na fase aguda é pouco comum ${ }^{11}$.

$\mathrm{Na}$ Amazônia brasileira, área considerada de baixa endemicidade, já se encontra bem estabelecido o ciclo enzoótico de DC, entre mamíferos e triatomíneos silvestres da região, que se apresentam com elevado índice de infecção natural pelo $T$. cruzi ${ }^{8}{ }^{9}$. Associado a isto, a exposição crescente do homem ao ciclo silvestre da doença incrementa a casuística da doença humana nesta região.

Os primeiros casos humanos autóctones de DC na Amazônia surgiram a partir de 1966, com o relato de três casos por Shaw et al, em Belém do Pará. Tornaram-se casos importantes por terem sido detectados simultaneamente, na mesma residência e na ausência de triatomíneos antropofílicos domiciliares. Os autores sugeriram, na ocasião, a possibilidade de transmissão da doença por meio diverso do habitual, aventando a hipótese de infecção por via oral, a partir da contaminação alimentar com fezes de barbeiro infectado ${ }^{16}$; posteriormente, esta hipótese foi testada experimentalmente, confirmando a transmissão oral em animais de laboratório ${ }^{12}$.

\footnotetext{
1. Instituto Evandro Chagas da Fundação Nacional de Saúde. 2. Instituto do Coração, Belém, PA. Apoio financeiro: Instituto Evandro Chagas/FUNASA e Instituto do Coração/Belém

Endereço para correspondência: Dra. Ana Yecê das Neves Pinto. Programa de Doença de Chagas/Instituto Evandro Chagas/FUNASA. Rod. BR 316 km 7, Marco, 67030-070 Ananindeua, PA.

Tel: 91 214-2150/214-2107; Fax:91 214-2043

e-mail:ayece@iec.pa.gov.br
}

Recebido para publicação em 24/2/2000. 
Até o momento já foram descritos aproximadamente 148 casos, sendo 121 considerados agudos, dos quais 5 evoluíram para o óbito e 27 de classificação não especificada, distribuídos em toda a região Amazônica brasileira, incluindo os Estados do Pará, Amazonas, Acre, Amapá, Maranhão (considerando-se a Amazônia Legal) e Rondônia. Atualmente o Estado que mais tem notificado casos é o Pará, somando um total de 71 casos, que inclui casos ocorridos em 17 microepidemias familiares já descritas ${ }^{19}$.

As características clínicas mais evidenciadas nos casos já descritos são febre intermitente acompanhada de artralgias, calafrios, mialgias e em muitos casos rush cutâneo, de aparecimento, em média, após cinco a seis dias do início do quadro ${ }^{561720}$. Além disso, há o relato de pacientes com manifestações de cardite aguda, com referência à dispnéia aos pequenos e médios esforços, palpitações, taquicardia, edema de membros inferiores e hepatomegalia. Estes quadros variam desde acometimentos leves até formas graves, com relato de três óbitos por insuficiência cardíaca refratária6 132021.

A partir da implantação de um Sistema de Vigilância Entomológica e Epidemiológica de DC na Amazônia brasileira em 1993, faz-se necessário conhecermos cada vez mais o comportamento da infecção chagásica em humanos da região, a fim de subsidiarmos o sistema de vigilância. Objetivouse, com este trabalho, mostrar os principais achados clínicos, eletrocardiográficos e ecocardiográficos relativos ao acometimento cardíaco encontrados em pacientes no período inicial de DC em mais um episódio de microepidemia familiar na Amazônia brasileira.

\section{MATERIAL E MÉTODOS}

Foram estudados 13 pacientes, pertencentes a quatro núcleos familiares, diagnosticados em novembro de 1998. A investigação epidemiológica evidenciou tratar-se de casos indubitavelmente autóctones, procedentes do município de Abaetetuba, no Estado do Pará, situado a $100 \mathrm{~km}$ da capital.

Após o diagnóstico dos dois primeiros casos através do exame de imunofluorescência indireta com pesquisa de lgM anti-T.cruzi positiva, mais nove doentes, todos relacionados familiar ou geograficamente a esses dois primeiros casos, procuraram espontaneamente 0 Instituto Evandro Chagas (IEC), tendo sido também diagnosticados. Passado um mês após os primeiros diagnósticos, duas pessoas do sexo feminino, empregadas domésticas na residência de uma das famílias acometidas, procuraram o Serviço e também tiveram seu diagnóstico firmado, totalizando 13 pessoas referentes ao mesmo surto.

Os dois primeiros casos detectados, um casal que iniciou quadro febril em 11 de outubro de 1998, tiveram seu diagnóstico inicial de febre tifóide e foram tratados com cloranfenicol. Como não houvesse melhora dos sinais e sintomas, foram encaminhados para o Serviço de Bacteriologia do IEC. O médico assistente deste Serviço, com experiência acumulada no diagnóstico de casos de DC oriundos do interior do Estado, encaminhou-os ao Programa de doença de Chagas/ IEC. Foram então firmados os diagnósticos de período inicial de DC autóctone em 10 de novembro do mesmo ano, pela presença de anticorpos IgM e quadro clínico compatível de duração menor que 28 dias.

A partir destes dois casos, o restante das pessoas envolvidas no episódio foram diagnosticadas paulatinamente, visto que todas apresentavam história muito semelhante de febre intermitente com início entre os dias 11 e 16 de outubro de 1998, acompanhada de mialgias intensas, e edema de face e membros inferiores com duração entre 15 e 20 dias de doença.

Os dados demográficos referentes ao grupo familiar, idade, sexo, além das informações acerca do diagnóstico parasitológico e sorológico de cada doente podem ser vistos na Tabela 1.

Os doentes foram submetidos a tratamento específico a partir de 11 de novembro de $1998 \mathrm{com}$ benzonidazol (Rochagan ${ }^{\circledR}$ )a dose de 5 a $7 \mathrm{mg} / \mathrm{kg} / \mathrm{dia}$ durante 60 dias, cuja apreciação será feita posteriormente.

O diagnóstico foi estabelecido pela evidência de anticorpos IgM anti-T.cruzi e a presença de parasitas circulantes visibilizados pelo xenodiagnóstico.

Todos os casos foram submetidos à avaliação clínica completa incluindo anamnese e exame físico e realizaram exames laboratoriais específicos: hemoscopia direta, esfregaço sanguíneo corado pelo método de Walker, Quantitative Buff Coat (QBC), xenodiagnóstico e imunfluorescência indireta para pesquisa de anticorpos IgM e IgG anti-Trypanosoma cruzi.

A avaliação cardiológica foi feita por médico cardiologista tendo sido realizados ecocardiograma bidimensional com mapeamento de fluxo em cores (ECO), conforme preconiza a Sociedade Americana de Ecocardiografia ${ }^{15}$ e exame eletrocardiográfico ECG convencional e dinâmico (Holter de 24 horas), na chegada do doente (antes do início do tratamento) ou, no máximo, até quatro dias após o mesmo.

Foram descritas as apresentações clínicas e os achados ecocardiográficos e eletrocardiográficos dos casos para eventual comparação com casos anteriormente descritos. Os dados epidemiológicos relativos à forma de transmissão, não serão discutidos no momento. 
Tabela 1 - Dados demográficos conforme grupos familiares, referentes a treze pacientes portadores de doença de Chagas aguda e principais resultados de exames parasitológicos e sorológicos correspondentes ao início do tratamento. Abaetetuba/PA, 1999.

\begin{tabular}{|c|c|c|c|c|c|c|c|c|}
\hline \multirow{3}{*}{ Grupo } & \multirow{3}{*}{ Paciente } & \multirow{3}{*}{ Sexo } & \multirow{3}{*}{$\begin{array}{l}\text { Idade } \\
\text { (anos) }\end{array}$} & \multicolumn{5}{|c|}{ Exames } \\
\hline & & & & \multirow[t]{2}{*}{ Esfregaço } & \multirow[t]{2}{*}{ QBC } & \multirow[t]{2}{*}{ Xenodiagnóstico } & \multicolumn{2}{|c|}{$\mathrm{IFI}$} \\
\hline & & & & & & & $\lg M$ & $\lg G$ \\
\hline \multirow[t]{5}{*}{$1^{\circ}$} & BIPS & $\mathrm{F}$ & 38 & negativo & negativo & positivo & $1 / 40$ & $1 / 640$ \\
\hline & RNM & $\mathrm{F}$ & 79 & negativo & negativo & positivo & $1 / 160$ & $1 / 160$ \\
\hline & MMFR & $\mathrm{F}$ & 42 & negativo & negativo & positivo & $1 / 320$ & $1 / 40$ \\
\hline & JCFR & $\mathrm{F}$ & 18 & negativo & negativo & positivo & $1 / 640$ & $1 / 320$ \\
\hline & JBFF & $\mathrm{F}$ & 26 & negativo & negativo & positivo & $1 / 160$ & $1 / 80$ \\
\hline \multirow[t]{3}{*}{$2^{0}$} & JSFQ & M & 27 & negativo & negativo & positivo & $1 / 160$ & $1 / 80$ \\
\hline & JFFF & M & 28 & negativo & negativo & positivo & $1 / 40$ & $1 / 160$ \\
\hline & LMPF & $\mathrm{F}$ & 43 & negativo & negativo & positivo & $1 / 640$ & $1 / 80$ \\
\hline \multirow[t]{3}{*}{$3^{\circ}$} & JBPF & M & 11 & negativo & negativo & positivo & $1 / 80$ & $1 / 640$ \\
\hline & SPF & M & 14 & negativo & negativo & positivo & $1 / 160$ & $1 / 80$ \\
\hline & AJF & M & 42 & negativo & negativo & positivo & $1 / 160$ & $1 / 80$ \\
\hline \multirow[t]{2}{*}{$4^{\circ}$} & ALF & $\mathrm{F}$ & 56 & negativo & Positivo & positivo & $1 / 320$ & $1 / 160$ \\
\hline & MJSF & M & 63 & negativo & negativo & positivo & negativo & 1320 \\
\hline
\end{tabular}

Fonte: Programa de Doença de Chagas/Instituto Evandro Chagas. 1999

\section{RESULTADOS}

A Tabela 2 evidencia os principais achados clínicos detectados no início do tratamento ou referidos no início do quadro clínico e à época do diagnóstico. Cumpre assinalar que nenhum paciente apresentou quaisquer sinais de porta de entrada da infecção.

As manifestações cardiovasculares foram significativas e incluíram palpitações, dispnéia aos pequenos e médios esforços (sem referências a dispnéia paroxística noturna) e edema de membros inferiores (de origem não caracterizada). A taquicardia, manifestada por três doentes durante o exame clínico, variou entre 100 a 120 batimentos por minuto.
No exame físico especial, os principais achados em relação à ausculta cardíaca podem ser vistos na Tabela 3.

Apenas três pacientes realizaram $\mathrm{Rx}$ de tórax, dos quais, um apresentou resultado normal e, em dois pacientes foi evidenciado discreto aumento de área cardíaca.

As alterações encontradas nos exames ecocardiográficos e eletrocardiográficos, realizados em média após 45 dias de início da sintomatologia, podem ser vistas na Tabela 4 .

Tabela 2 - Principais achados clínicos no primeiro dia de tratamento de treze pacientes portadores de doença de Chagas aguda. Abaetetuba/PA, 1999

\begin{tabular}{lcc}
\hline \multirow{2}{*}{ Sinais e/ou sintomas } & \multicolumn{2}{c}{ Freqüência } \\
\cline { 2 - 3 } & $\mathrm{n}^{\circ}$ & \multicolumn{1}{c}{} \\
\hline Febre intermitente & 13 & 100,0 \\
Edema de MMII & 10 & 76,9 \\
Cefaléia & 9 & 69,2 \\
Mialgias & 7 & 53,9 \\
Artralgias & 7 & 53,9 \\
Cansaço aos esforços & 4 & 30,8 \\
Taquicardia & 4 & 30,8 \\
Exantema máculo-papular & 3 & 23,1 \\
Palpitações & 2 & 15,4 \\
Nódulos subcutâneos & 2 & 15,4 \\
Esplenomegalia & 1 & 7,7 \\
Enfartamento ganglionar & 0 & 0,0 \\
\hline Fonte: Programa de Doença de Chagas/Instituto Evandro Chagas. 1999
\end{tabular}

Fonte: Programa de Doença de Chagas/Instituto Evandro Chagas. 1999 
Dos 13 pacientes, observou-se que em sete $(53,9 \%)$ o ECG encontrava-se normal. No ECG dinâmico, cinco dos sete pacientes com ECG normal apresentaram alguma alteração. As extra-sístoles supraventriculares (ESSV) e/ou ventriculares (ESV), evidenciadas principalmente ao Holter, estiveram presentes em $8(61,5 \%)$ dos 13 doentes; contudo, a maioria destas alterações não tinha relação com a sintomatologia referida pelos pacientes. Bloqueios de ramo direito (BRD) e bloqueios átrio-ventriculares (BAV) de $1^{\circ}$ e $2^{\circ}$ graus foram encontrados em $4(30,8 \%)$ dos 13 doentes. As alterações menos freqüentes

Tabela 3 - Principais achados da ausculta cardíaca de treze pacientes portadores de cardiopatia chagásica aguda. Abaetetuba/PA, 1999

\begin{tabular}{lrrr} 
Achados na ausculta cardíaca & \multicolumn{2}{c}{ Freqüência } \\
\cline { 2 - 3 } & \multicolumn{2}{c}{$\mathrm{n}$} & $\%$ \\
\hline BCNF RCR em 2 t & 7 & 53,9 \\
BCNF com desdobramento de B2 & 3 & 23,1 \\
BCNF com desdobramento de B2 ? Sopro sistólico? & 1 & 7,7 \\
Sopro sistólico em foco aórtico & 1 & 7,7 \\
BCNF RCI ${ }^{2}$ Extra sístoles (2/min) & 1 & 7,7 \\
\hline
\end{tabular}

${ }^{1}$ Bulhas cardíacas normofonéticas, ritmo cardíaco regular em dois tempos

${ }^{2}$ Ritmo cardíaco irregular

Fonte: Programa de Doença de Chagas/Instituto Evandro Chagas. 1999

foram: QRS de baixa voltagem e as alterações difusas a repolarização ventricular, presentes em 3 (23,1\%) dos 13 traçados. Ocorreu uma manifestação de taquicardia paroxística ventricular (TPV) nãosustentada sem repercussões.
Chamam a atenção dois achados no ecodopplercardiograma: derrame pericárdico e uma imagem sugestiva de formação aneurismática em dois pacientes distintos.

Tabela 4 - Achados de eletrocardiograma simples e 24 horas e ecocardiograma, realizados em 13 pacientes com doença de Chagas aguda. Abaetetuba, 1999.

\begin{tabular}{|c|c|c|}
\hline Paciente & Paciente ECG e/ ou Holter & $\begin{array}{l}\text { Ecocardiograma e Doppler } \\
\text { Vascular }\end{array}$ \\
\hline BIPS & $\begin{array}{l}\text { ECG: taquicardia sinusal; alterações difusas de repolarização } \\
\text { Holter: Normal }\end{array}$ & Normal \\
\hline RNM & $\begin{array}{l}\text { ECG: Normal } \\
\text { Holter: } 16 \text { ESSV com períodos de salvas; } 2 \text { ESSV. Condução AV normal. }\end{array}$ & Normal \\
\hline MMFR & $\begin{array}{l}\text { ECG: Normal } \\
\text { Holter: Condução AV normal; } 1 \text { ESSV isolada. }\end{array}$ & Aumento leve de $\mathrm{AE}$. \\
\hline JCFR & $\begin{array}{l}\text { ECG: Normal } \\
\text { Holter: BAV } 2^{\circ} \text { grau; fenômeno Weckenbach; } 2 \text { ESV monomórficas isoladas }\end{array}$ & Aumento leve de ve? \\
\hline $\begin{array}{l}\text { JBFF } \\
\text { JSFQ }\end{array}$ & $\begin{array}{l}\text { ECG - Bloqueio de Ramo D } \\
\text { ECG: BAV } 1^{\circ} \text { grau; Alentecimento de condução Ramo D; ADR (sugestiva } \\
\text { de isquemia subepicárdica) em parede anterior. } \\
\text { Holter: } 2 \text { ESSV isoladas, } 16 \text { ESV pmf isoladas, } 2 \text { epis. TPV não sustentada. }\end{array}$ & $\begin{array}{l}\text { Normal } \\
\text { Normal }\end{array}$ \\
\hline $\begin{array}{l}\text { JFFF } \\
\text { LPF }\end{array}$ & $\begin{array}{l}\text { ECG e Holter: Normais } \\
\text { ECG: Normal } \\
\text { Holter: Normal }\end{array}$ & $\begin{array}{l}\text { Normal; mínimo refluxo mitral } \\
\text { Dilatação leve VE; } \\
\text { Mínimo refluxo mitral }\end{array}$ \\
\hline JBPF & $\begin{array}{l}\text { ECG: QRS de baixa voltagem. ADR } \\
\text { Holter: Normal }\end{array}$ & Normal \\
\hline SPF & $\begin{array}{l}\text { ECG: ADR/ Holter: } 34 \text { ESSV isoladas, } 31 \text { ESV polimórficas (pmf); } 3 \\
\text { episódios de taquicardia ventricular não acelerada }\end{array}$ & Normal; Mínimo refluxo tricúspide \\
\hline ALF & $\begin{array}{l}\text { ECG: Normal } \\
\text { Holter: Ritmo sinusal, } 2 \text { ESV isoladas, } 498 \text { ESV pmf. com bigeminismo }\end{array}$ & Disfunção diastólica mitral \\
\hline MJSF & $\begin{array}{l}\text { ECG: Normal } \\
\text { Holter Ritmo sinusal; } 4 \text { ESSV isoladas, } 1271 \text { ESSV pmf. c/ trigeminismo }\end{array}$ & $\begin{array}{l}\text { Dilatação leve VE; leve } \\
\text { hipocinesia apical }\end{array}$ \\
\hline AJF & $\begin{array}{l}\text { ECG: Complexo QRS baixa voltagem, onda T negativa ínfero-lateral } \\
\text { Holter: BAV } 1^{\circ} \text { grau, BR transitório; } 14 \text { ESSV isoladas, } 38 \text { ESV pmf. }\end{array}$ & $\begin{array}{l}\text { Refluxo mitral; derrame pericárdico } \\
\text { leve; Insufic. Mitral }\end{array}$ \\
\hline
\end{tabular}

Fonte: Programa de Doença de Chagas/Instituto Evandro Chagas. 1999 


\section{DISCUSSÃO}

A Amazônia brasileira, antes área indene de doença de Chagas, tem hoje computados mais de 200 casos da mesma em humanos e vem desenhando sob nossos olhos um quadro semelhante àquele ocorrente em áreas endêmicas, porém com peculiaridades muito próprias já evidenciadas, e em contínua caracterização, principalmente em seus aspectos clínicos e epidemiológicos.

Quantos aos aspectos epidemiológicos, a detecção de casos agudos ocorrendo sob a forma de microepidemias familiares de provável transmissão por alimentos crus ou mal cozidos constitui uma destas peculiaridades, funcionando como marcador de potencialidade da parasitose nesta região ${ }^{4}{ }^{17}$, e possibilitando o uso do termo Tripanosomíase Amazônica de forma a diferenciá-la da entidade clínica classicamente descrita por Chagas.

Em relação ao aspecto clínico, segundo Dias, as manifestações cardiovasculares da DC aguda foram muito bem estudadas nos trabalhos fundamentais de Carlos Chagas em 1910, cabendo ao grupo de estudos formado no Centro Emanuel Dias (FIOCRUZ, Bambuí, MG) ratificar as observações pioneiras e subsidiar 0 quadro clínico da cardiopatia chagásica aguda em áreas endêmicas. Como exemplo, um estudo de 313 casos de Bambuí revelou que o acometimento cardiovascular na DC aguda é muito freqüente especialmente nos indivíduos de baixa idade, fato já amplamente frisado por Chagas e consonante com vários estudos experimentais ${ }^{11}$.

Na Amazônia brasileira, além dos quadros comuns febris já descritos, são frequentes os relatos de pacientes com manifestações de cardite aguda, caracterizados por dispnéia aos pequenos e médios esforços, palpitações, taquicardia, edema de membros inferiores decorrentes ou não de acometimento cardíaco e hepatomegalia. Infelizmente, por dificuldades técnicas das localidades de origem dos casos, a maioria deles não apresenta registro documentado do comprometimento cardíaco (minimamente um traçado eletrocardiográfico, por exemplo). Assim, dos casos anteriores ao presente estudo, existem referências a alterações da repolarização ventricular e hipertrofia de ventrículo esquerdo em dois $\operatorname{casos}^{5}$, sendo o primeiro achado, também incidente nos pacientes ora estudados. Um levantamento feito em 1994 a partir de 20 pacientes com DC aguda revelou alterações eletrocardiográficas em metade dos pacientes, incluindo aumento do intervalo PR, baixa voltagem de QRS e anormalidades na onda $T^{6}$.

Em áreas endêmicas, é interessante a confrontação radiológica-eletrocardiográfica, verificando-se entre 70 a $80 \%$ de indivíduos apresentando algum aumento de área cardíaca frente a mais de $50 \%$ de eletrocardiogramas normais, revelando uma relativa benignidade da cardiopatia chagásica aguda (CCA) e seu elevado limiar clínico e eletrocardiográfico ${ }^{10}$. Nestas áreas, refere-se que a evolução da CCA é geralmente homogênea e consensualmente de prognóstico reservado em crianças de baixa idade, pela presença de insuficiência cardíaca congestiva (ICC), com letalidade em torno de $5 \%$ e evolução rápida para o choque, em quadros já muito bem caracterizados por Chagas em 1916. Nos sobreviventes, o quadro em geral evolui para regressão total, paralelamente à negativação do exame parasitológico direto.

$\mathrm{Na}$ Amazônia brasileira, três casos de ICC por miocardiopatia chagásica aguda foram descritos, sendo que um paciente de cinco anos de idade evoluiu para o óbito ${ }^{21}$. No presente estudo, apesar da maioria dos doentes apresentarem alguma sintomatologia cardíaca, todos evoluiram satisfatoriamente; mesmo o paciente AJF que durante internamento hospitalar fez bradiarritmia grave (tendo sido aventada a possibilidade de implante de marcapasso), evoluiu de forma excelente após início da terapêutica específica, apesar de ter mantido, até o momento, BAV de $1^{\circ}$ grau.

Nos pacientes estudados, o ECG convencional mostrou-se normal em $53,9 \%$ dos pacientes e o ECG dinâmico em $38,5 \%$.

A ecodopplercardiografia que possibilita melhor avaliação da evolução da cardiopatia chagásica, constitui procedimento de escolha para detecção de lesões aneurismáticas. Um estudo recente, em área endêmica (Virgem da Lapa/MG), evidenciou 18,8\% de aneurismas de VE utilizando o ECO em 298 pacientes chagásicos crônicos ${ }^{2}$.

Com este exame, observou-se um porcentual de normalidade de $53,9 \%$ dos nossos casos, porém, a detecção de um provável aneurisma de ponta de VE interrogado suscita alguma discussão, considerandose a precocidade do aparecimento da lesão. A paciente que manifestou esta alteração ao ECO é uma das pessoas que prestava serviços domésticos para uma das famílias envolvidas no episódio, e que na época do diagnóstico dos primeiros casos, ambas não tiveram condições financeiras de vir até Belém, onde está situado o IEC. Assim, só efetuaram esta visita cerca de dois meses após o quadro agudo quando já se encontravam afebris e oligossintomáticas. $O$ dado cronológico do diagnóstico tardio e a evidência ecocardiográfica, em uma destas pacientes, de lesão indicadora de fase crônica ${ }^{14}$, dificultam a classificação de ambas como período inicial da DC, diferentemente dos outros casos. Todavia, os argumentos para inclusão das mesmas como período inicial são fortes, sendo um laboratorial: a detecção de anticorpos da classe IgM; outro clínico: presença de sintomas e 
sinais residuais como mialgias e edema maleolar, os quais por si só são inespecíficos da DC, porém, são indicadores de doença aguda recente levando-se em consideração que estas pacientes apresentaram síndrome febril compatível cronológica e clinicamente com os casos concomitantes.

Os autores sugerem que estas duas pacientes se encontravam, na época de seu diagnóstico, em uma fase de transição entre o período inicial e a fase crônica inicial, coadunando fenômenos característicos de ambas as fases.

As ESSV e/ou ESV evidenciadas principalmente ao Holter, não tiveram relação com sintomatologia referida pelos pacientes, sendo poucas as descrições destas alterações em casos agudos ocorridos em áreas endêmicas; eventualmente, são alterações também encontradas em populações de indivíduos não-chagásicos.

Por outro lado, são freqüentes as descrições dos distúrbios de condução relacionados a DC aguda ou crônica nas áreas endêmicas, em conseqüência do acometimento cardíaco difuso determinado pelo T.cruzi, e à semelhança desta descrição, os casos ora apresentados revelaram algum tipo de disfunção da condução átrio-ventricular $(30,8 \%$ dos doentes) $\mathrm{BRD}, \mathrm{BAV}$ de $1^{\circ}$ e $2^{\circ}$ graus - com poucas repercussões clínicas e resolução satisfatória após tratamento, estando programado controle eletrocardiográfico.

As alterações menos frequentemente encontradas nos pacientes de Abaetetuba (QRS e ADVR, são descritas na literatura como as irregularidades mais comumente registradas eletrocardiograficamente nas áreas endêmicas em casos agudos ${ }^{1}$. Estas alterações estão relacionadas, freqüentemente, a processos inflamatórios do miocárdio (miocardites), como também às pericardites, principalmente aquelas que cursam com derrame pericárdico, a exemplo do que ocorreu com um dos pacientes estudados, o qual apresentou discreto derrame pericárdico ao ecocardiograma e complexo QRS de baixa voltagem ao ECG.
A taquicardia sinusal, relatada comumente nas áreas endêmicas, foi evidenciada no momento do exame clínico em três casos de nossa amostra, com freqüências cardíacas variando entre 100 a 120 batimentos por minuto. $O$ encontro pouco freqüente de taquicardia sinusal pode ser conseqüência de exames clínicos relativamente tardios. TVNS foi observada em um paciente (dois episódios) sem repercussão.

Os achados revelam comprometimento cardíaco agudo, com evidências de miocardiopatia no grupo estudado e alterações importantes no sistema de condução do coração, revelando similaridade com a descrição da doença em áreas consideradas endêmicas. As peculiaridades relativas à presença de BRD como sinal de mau prognóstico, e lesão aneurismática cardíaca de aparecimento precoce nesta pequena amostra de doentes, apontam para a especial atenção que deve ser dada aos portadores da infecção chagásica aguda em nossa região.

O ECG demonstrou ser um exame simples e efetivo na detecção de distúrbios apresentados por estes pacientes, mesmo considerando que metade deles não apresentava alterações detectáveis no momento do exame clínico. Devemos considerar, entretanto, que a maioria dos doentes já apresentava algum grau de resolutividade própria da história natural da infecção chagásica humana no momento do exame, realizado em média 45 dias após início do quadro agudo.

O ECG dinâmico, como seria de se esperar, foi mais sensível para detectar as alterações, sendo seu custo um fator proibitivo na indicação de rastreamento diagnóstico de portadores de DC aguda em nossa região.

A identificação precoce de lesões sem alteração no ECG convencional já foi demonstrado anteriormente ${ }^{3}$, por meio da cineangiocoronariografia, sendo corroborado nesta pequena amostra de doentes, ao evidenciar pelo ECO uma imagem sugestiva de lesão aneurismática classicamente descrita em chagásicos crônicos.

\title{
AGRADECIMENTOS
}

\author{
A Jovilson de Jesus Viana e Mário Barbosa (CR/Fundação Nacional de Saúde).
}

\section{REFERÊNCIAS BIBLIOGRÁFICAS}

1. Amato Neto V, Shikanai Yasuda MA, Amato VS. Doença de Chagas Aguda. In: Dias JCP Coura JR (eds) Clínica e Terapêutica da Doença de Chagas. Uma abordagem prática para o clínico geral. Fundação Osvaldo Cruz, Rio de Janeiro, p.127-133, 1997.

2. Borges-Pereira J, Xavier SS, Pirmez C, Coura JR. Avaliação ecocardiográfica de chagásico em área endêmica. Revista da Sociedade Brasileira de Medicina Tropical 30 (supl I):67, 1997.
3. Carrasco HA, Barboza JS, Inglessis G, Fuenmayor A, Molina C. Left ventricular cineangiography in Chagas disease: detection of early myocardial damage. American Heart Journal 3:595602, 1982.

4. Coura JR. Mecanismo da infecção chagásica ao homem por via oral. Revista da Sociedade Brasileira de Medicina Tropical 30 (supl I):45, 1997. 
5. Crescente A, Valente SAS, Valente VC, Araújo JS. Ocorrência de 4 casos agudos de Doença de Chagas na Vila de Icoaraci, PA. In: Anais XXVIII Congresso da Sociedade Brasileira de Medicina Tropical. Belém, p.29, 1992.

6. Crescente JAB, Pinheiro NCN, Valente SAS, Rodrigues RMV, Rebelo RR, Ayres SFG, Cavalcante MC. Análise clínica e epidemiológica de 20 casos autóctones de Doença de Chagas Aguda ocorridos no período de janeiro de 1988 a dezembro de 1992, Estado do Pará. Revista da Sociedade Brasileira de Medicina Tropical 27 (supl I):182, 1994.

7. Deane LM. Animal reservoirs of Trypanosoma cruzi. Revista Brasileira de Malariologia e Doenças Tropicais 16: 27-48, 1964.

8. Deane LM. Tripanosomídeos de mamíferos da região Amazônica IV. Hemoscopia e xenodiagnóstico de animais silvestres da estrada Belém-Brasília. Revista do Instituto de Medicina Tropical de São Paulo 9:143-148, 1967.

9. Deane MP, Lenzi HL, Jansen AM. Trypanosoma cruzi: vertebrate and invertebrate cycles in the same mammal host, the opossum Didelphis marsupialis. Memórias do Instituto Oswaldo Cruz 79:513-515, 1984.

10. Dias JCP. Doença de Chagas em Bambuí, MG, Brasil. Estudo clínico-epidemiológico a partir da fase aguda, entre 1940 e 1982. Tese. Universidade Federal de Minas Gerais. Belo Horizonte, MG, 1982.

11. Dias JCP. História natural. In: Cançado JR, Chuster M (eds), Cardiopatia Chagásica. Fundação Carlos Chagas de Pesquisa Médica. Belo Horizonte, p.9-113, 1985.

12. Lainson R, Shaw JJ, Fraiha H, Miles MA, Draper CC. Chagas' Disease in the Amazon Basin: 1 - Trypanosoma cruzi infections in silvatic mammals, triatomine bugs and man in the State of Pará, north Brazil. Transactions Royal Society of Tropical Medicine and Hygiene 73:193-204, 1979.

13. Pinto AYN, Valente VC, Valente SAS, Lima JA, Araújo JA. Apresentação de um caso autóctone agudo de Doença de
Chagas em Cachoeira do Arari, estado do Pará. Revista Brasileira de Parasitologia Veterinária 2 (supl I):98, 1993.

14. Rocha MOC, Ribeiro ALP, Torres RM, Vaz-Tostes VT. Métodos não invasivos de análise funcional cardíaca. In: Dias JCP e Coura JR (eds). Clínica e Terapêutica da Doença de Chagas. Uma abordagem prática para o clínico geral. Fundação Osvaldo Cruz, Rio de Janeiro, 1997.

15. Schiller NB, Shah PM, Crawtord M, Mana A, Devereux R, Feigenbaum H, Gutfesell H, Tajik AS. Recomendations for quantitation of the left ventricle by two-dimensional echocardiography. Journal of American Society Echocardiography 2:358-365, 1989.

16. Shaw JJ, Lainson R, Fraiha H. Considerações sobre a epidemiologia dos primeiros casos autóctones de Doença de Chagas registrados em Belém, Pará, Brasil. Revista de Saúde Pública 3:153-157, 1969.

17. Silveira, AC. Situação do controle da transmissão vetorial da Doença de Chagas no Brasil. Revista da Sociedade Brasileira de Medicina Tropical 30 (supl I):42, 1997.

18. Valente SAS e Valente VC. Epidemiologia e transmissão da Doença de Chagas na Amazônia Brasileira. Revista da Sociedade Brasileira de Medicina Tropical 30 (supl I):51, 1997.

19. Valente SAS, Valente VC, Frahia Neto H. Considerations on the Epidemiology and Transmission of Chagas Disease in the Brasilian Amazon. Memórias do Instituto Oswaldo Cruz 94 (supl I):395-398, 1999.

20. Valente SAS, Valente VC, Silva FM, Ferreira FM, Santos TCM, Moura E, Uchoa AD. Registro de Doença de Chagas Agudo em Sena Madureira, Estado do Acre. Revista da Sociedade Brasileira de Medicina Tropical 27 (supl I):169, 1994.

21. Viana S, Farias E, Lima F, Batista L, Vieira A, Silva L, Lobato C, Nascimento S, Chalub S. Doença de Chagas no estado do Acre, registro de 3 casos de Miocardiopatia Chagásica Aguda autóctone no Município de Rio Branco, 1993. Revista da Sociedade Brasileira de Medicina Tropical 27 (supl I):77, 1994. 